\title{
Enabling High Aspect Ratio Nano-hole Metal Filling by Employing Supercritical Carbon Dioxide Electrochemical Deposition
}

\author{
Wen-Ta Tsai*, Jun-Jie Yang, Chi-Lin Cheng \\ Department of Materials Science and Engineering \\ National Cheng Kung University \\ Tainan, Taiwan \\ wttsai@mail.ncku.edu.tw
}

\section{Extended Abstract}

In supporting structural integrity of a miniature component, or for providing electric conduction of a through-silicon via (TSV) in three-dimensional integrated circuits (3D-IC), micro- or nano-hole filling with metallic material becomes increasing important in various micro-electrical mechanical systems (MEMSs) [1-4] and in the advanced semiconductor industry [5]. Although there are several methods available for filling micro- or nano-holes with various types of materials, most of them still suffer incomplete filling and void (or defect) formation. When electrodeposition technique is applied, the lack of wettability of the electrolyte inside via or hole and/or the gas bubble formation are always of great concern. To overcome such problems, the use of an electrolyte incorporating emulsified supercritical carbon dioxide $\left(\mathrm{sc}-\mathrm{CO}_{2}\right)$ fluid has been proposed. In our previous study, the filling of micro-sized holes (about $50 \mu \mathrm{m}$ diameter and an aspect-ratio of 8 ) by electrodeposition of $\mathrm{Ni}$ and Ni-P employing sc- $\mathrm{CO}_{2}$ bath could be achieved [6]. The advantages of using supercritical carbon dioxide containing electrolyte are mainly attributed to its unique characteristics, such as liquid-like density, low surface tension, high diffusivity, low viscosity, and high hydrogen gas solubility, etc.

In this study, an anodic aluminum oxide (AAO) template containing numerous high aspect ratio nano-sized holes (an average diameter of $130 \mathrm{~nm}$ and an aspect-ratio about 20) was used as the cathode. The hydrophobicity/hydrophilicity of the AAO template was adjusted before $\mathrm{Cu}$ electrodeposition. Electrodeposition of $\mathrm{Cu}$ was carried out in an emulsified $\mathrm{sc}-\mathrm{CO}_{2}$ bath containing cupric sulfate aqueous solution as the precursor. $\mathrm{Cu}$ deposition into the nano-holes was performed at an apparent constant current density of $2 \mathrm{~A} / \mathrm{dm}^{2}$ for 5 minutes. After electrodeposition, the cross section morphology and chemical composition of the AAO cathode was examined by transmission electron microscopy (TEM) and energy dispersive spectroscopy (EDS) with the aid of a focused-ion-beam (FIB). The experimental results showed that electrodeposition employing sc- $\mathrm{CO}_{2}$ bath to fill $\mathrm{Cu}$ into nano-sized holes was successful and superior to that of conventional process carried out at ambient pressure, in terms of uniformity and deposition rate. The high solubility of hydrogen gas in sc- $\mathrm{CO}_{2}$ fluid is the key factor. Moreover, the advantage of $\mathrm{sc}-\mathrm{CO}_{2}$ electrodeposition is more pronounced for the AAO cathode exhibiting hydrophobic nature with respect to aqueous bath.

\section{References}

[1] Z. Wang, L. Wang, N. T. Nguyen, W. A. H. Wien, H. Schellevis, P. M. Sarro, and J. N. Burghartz, "Silicon Micromachining of High Aspect Ratio, High-Density Through-Wafer Electrical Interconnects for 3-D Multichip Packaging," IEEE Transactions on Advanced Packaging, vol. 29, pp. 615-622, 2006.

[2] J. K. Luo, D. P. Chu, A. J. Flewitt, S. M. Spearing, N. A. Fleck, and W. I. Milne, "Uniformity control of Ni thin-film microstructures deposited by through-mask plating," J. Electrochem. Soc., vol. 152, pp. C36-C41, 2005.

[3] N. Watanabe, M. Suda, K. Furuta, and T. Sakuhara, "Fabrication of micro parts using only electrochemical process," in Proceedings of the 14th IEEE International Conference on Micro Electro Mechanical Systems, pp. 143-146, 2001.

[4] J. Prokop, G. Finnah, J. Lorenz, V. Piotter, R. Ruprecht, and J. Hausselt, "Manufacturing process for high aspect ratio metallic micro parts made by electroplating on partially conductive templates," Microsystem Technologies, vol. 14, pp. 1669-1674, 2008.

[5] J. P. Gambino, S. A. Adderly, and J. U. Knickerbocker, "An overview of through-silicon-via technology and manufacturing challenges," Microelectronic Engineering, vol. 135, pp. 73-106, 2015. 
[6] C-Y. Li, J-J. Yang, W-T. Tsai, C-J. Lin, T-F. M. Chang, and M. Sone, "High aspect ratio micro-hole filling employing emulsified supercritical CO2 electrolytes," Journal of Supercritical Fluids, vol. 109, pp. 61-66, 2016. 